POS $\quad$ PROCEEDINGS

\title{
Peanut shaped structures in edge-on galaxies
}

\section{Giuseppe Aronica*}

AIRUB, Germany

E-mail: 'aronicaeastro. rub. dé

\section{Martin Bureau}

Columbia Astrophysics Laboratory

E-mail: 'bureaueastro. columbia.edú

\section{Evangelia Athanassoula}

Laberatoire d'Astrophyique de Marseille

E-mail: 'ía@oamp. fri

\section{Ralf-Jürgen Dettmar}

AIRUB, Germany

E-mail: 'dettmareastro.rub.dét

A photometric analysis of a sample of edge-on galaxies harboring a peanut shaped structure is presented. This structure is ideally suited to study the bar driven evolution of galaxies since it is slightly connected to the presence of vertical bar resonances.

For our study we use K-band imaging which is dominated by Population II stars and minimally affected by dust. Applying the unsharp masking filtering technique we are able to isolate the periodic orbit families at the origin of the particular morphology of a peanut shaped structure. The rearrangement of disk material in the vertical direction through the presence of a bar is evidenced by our study of vertical scale heights along the major axis of the galaxies. Fitting of generalized Gaussians (equivalent to a Sersic law) to the vertical surface brightness profiles shows a radial variation of scale heights reaching a maximum at positions where the peanut structure is most prominent.

Baryons in Dark Matter Halos

5-9 October 2004

Novigrad, Croatia

*Speaker. 


\section{Introduction}

In the context of spiral galaxy evolution the understanding of bulge formation processes are of central importance. Hierarchical galaxy formation theories describe the bulge formation by collapse of a primordial gas cloud into clumps and a subsequent merging of these clumps. The disk forms after this process.

Secular evolution of the disk is suggested as an alternative or additional scenario for bulge formation and evolution. The frequently observed box/peanut (b/p) shaped bulges in edge-on galaxies are supposed to be related to secular evolution effects in barred galaxies. These kind of bulges consist of a spheroidal bulge component in the centre of the disk and a superposed component of additional light forming a X-structure. Combes et al. [i1] describe in their simulations that vertical instabilities in bars, particularly the $2 / 1$ vertical Inner Lindblad Resonance, can induce the formation of the $\mathrm{X}$-structure and therefore lead to the formation of a box/peanut shaped bulge. The close connection of bars and the $\mathrm{b} / \mathrm{p}$ shape of bulges is observationally supported by the work of [2] $]$ ]

\section{Observations and data analysis}

Our analysis is based on a sample of 30 edge-on galaxies observed with the $2.3 \mathrm{~m}$ telescope at the Mount Stromlo Siding Springs Observatory in Australia. Since the aim of the observations was to trace the mass distribution of the galaxies, the NIR $K_{n}$-band filter was selected. The main part of the sample consists of galaxies (24) showing a B/PS structure in the central region, whereas the remaining 6 galaxies serve as a control sample. These galaxies show contrary to the others no B/PS structure. The analysis of the obtained photometric data was done using two approaches. In the first we applied the unsharp masking technique on the $K_{n}$-band imaging data to enhance the underlying substructure and in the second we applied a mulitparamter fit to the vertical light distribution of the $K_{n}$-band images. For the unsharp masking all galaxies were median filtered with a fixed range of region sizes to select after that by eye the size of the median filter best suited to enhance the present substructure in the galaxy (see Fig. ii i i). In the second approach for our analysis we have fitted the vertical light distribution of all galaxies of our sample with two fitting functions. Following the approach of [i-1] a generalized Gaussian is discribed by the following function $\Sigma_{g g}=\Sigma_{0} \cdot \exp \left[-\left(|z| / h_{z}\right)^{\lambda}\right]$. The 3 fitting parameters are the central surface brightness $\Sigma_{0}$, the scale height $h_{z}$ and the shape parameter $\lambda$ describing the shape of the function (see Fig. $2_{2}^{i}$ ). Using the generalized Gaussians makes the analysis independent of a choice fixing the form of the fitting function a priori. As a consequence we are independent of any assumption about the phyiscs leading to definite choice of the fitting function. Specially for the vertical structure of bars this is of great advantage since little is known in this field. In the case of disks the $\operatorname{sech}, \operatorname{sech}^{2}$ and $\exp$ are widely used fitting functions for the vertical light distribution To compare our results with an independent method we used also Gauss-Hermite polynoms. In this proceedings only the results of the generalized Gaussian approach are shown.

\section{Results}

In 18 cases out of 24 galaxies with a B/PS structure the vertical fitting of generalised gaussians shows an exponential vertical light distribution. For the rest of the sample the shape 

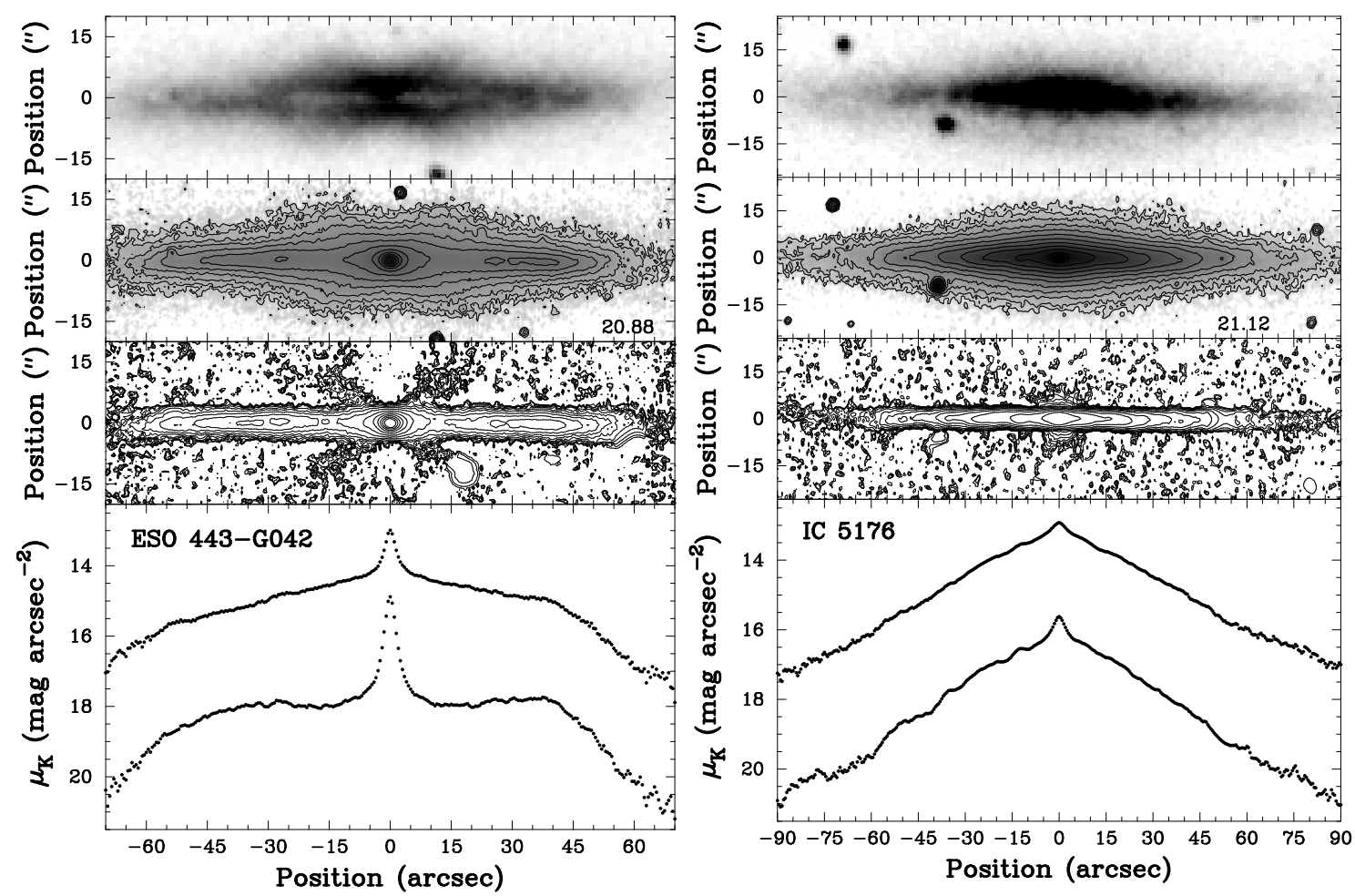

Figure 1: Images and surface brightness profiles for the galaxy ESO-443G042 and IC 5176. From the top to the bottom a DSS2 Red image of the galaxy is shown, a $K_{n}$-band image with contour plots equally spaced with in steps of $0.5 \mathrm{mag}$, the unsharp masked version of the $K_{n}$-band image. In the lowest panel the surface brightness profile along the major axis as well as the surface brightness profile obtained by summing all vertical light contribution are shown. The major axis surface brightness profile is the fainter one.

The two galaxies are chosen on the basis of their morpology. ESO-443G042 is good representative of the galaxies showing a B/PS structure and therefore harbouring a bar. The galaxy IC 5176 is fundamentaly

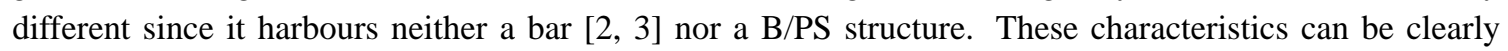
observed in the surface brightness plots, where components like a plateau, characteristic for bar signatures, is visible. In the case of an absence of a bar, like in IC 5176, no plateau can be detected.

parameter has values between a gaussian vertical distribution $(\lambda=2)$ and an exponential $(\lambda=1)$. When comparing the results with the values shown in [-1] ] the stronger pronounced minimum in the galactic center is most obvious. This means that in the simulations the transfer of material into the center of the galaxy is less then needed to obtain the deep and steep minimum as visible in the case of ESO 443-G042 (Fig. 'i2i). The exponential distribution is most pronounced at the outer edges of the bar signature. All galaxies showing a B/PS structure unveil in the unsharp masked images a complex substructure. The most prominent ones are $\mathrm{X}$ shaped structures which can be centered at the central regions of the galaxies or offcentered which means that the legs of the $\mathrm{X}$ do not cross the galactic center. These structures are strikingly similar to the orbits shown in ['5-1].]. Also in that case large $X$ structures in the center of model galaxies are visible. They are most pronounced in the case of a model with a strong bar.

The two approaches, unsharp masking and vertical fitting, show an interesting variety of structures and large qualitative concordance with simulations and orbital calculations. 

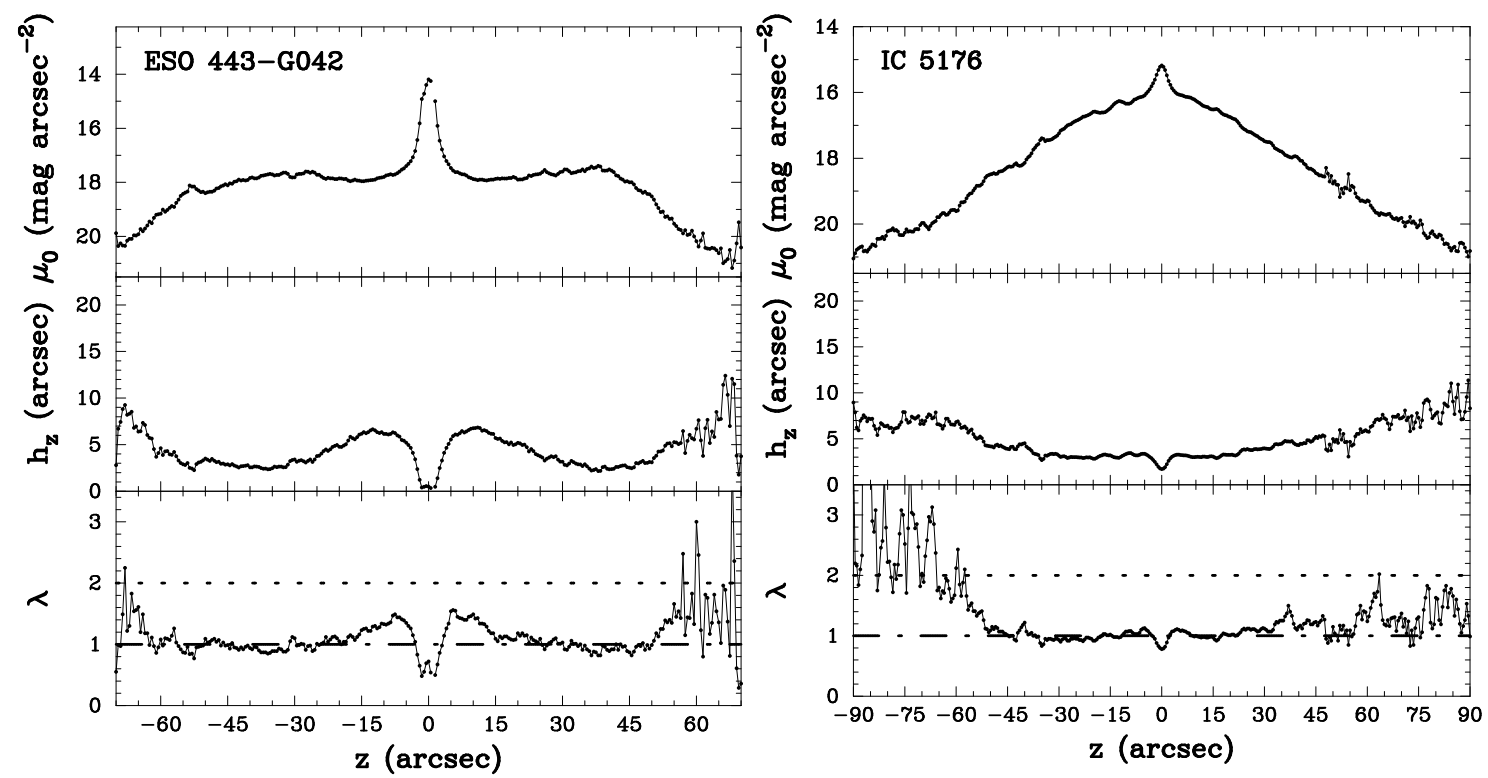

Figure 2: Results of the vertical fitting with a generalized Gaussian for ESO 443-G042 (left) and IC_5176 (right). In the top panel the fitted central surface brightness is plotted. The middel panel shows the fitted values of the vertical scale height in arcsecs whereas the bottom panel shows the shape parameter $\lambda$ obtained by the fitting. Differences in the structure of both galaxies are visible in the central regions. In the case of ESO 443-G042 the presence of a pronounced nuclear region and a B/PS structure can be observed. Within a region of $\approx 8$ arcsec around the center of the galaxy a nuclear component is present which can be distinguished by the shape parameter $\lambda$ having a global maximum in that region. Also the scale height reaches a minimum at the same location. Local minima to the left and to the right of that position reveal the presence of the B/PS structure. Interestingly the whole region dominated by the bar $(|R| \approx 55 \operatorname{arcsec})$ is characterised by a vertical distribution described by a pure exponential. Contrary to ESO 443-G042 the galaxy IC 5176 does not show a pronounced nucleus in the center, only a shallow minimum is dectectable in either the scale height plot as well as in the plot showing $\lambda$. Similar to the situation of ESO 443-G042 also in this case the vertical light distribution of the bar is exponential.

\section{References}

[1] F. Combes, F. Debbasch, D. Friedli, D. Pfenniger, Box and peanut shapes generated by stellar bars, 1990, A\&A, 233, 82

[2] B. Bureau, and K. Freeman, The Nature of Boxy/Peanut-Shaped Bulges in Spiral Galaxies, 1999, AJ, 118,126

[3] A.Chung, and M. Bureau, Stellar Kinematics of Boxy Bulges: Large-Scale Bars and Inner Disks, 2004, AJ, 127, 3192

[4] E. Athanassoula, and A. Misiriotis, Morphology, photometry and kinematics of N -body bars - I. Three mode ls with different halo central concentrations, 2002, MNRAS, 330, 35

[5] P. A, Patsis, Ch. Skokos, and E. Athanassoula, Orbital dynamics of three-dimensional bars: III. Boxy/Peanut edge-on profiles, 2002, MNRAS, 337, 578 